\title{
Reduction of the intensity noise from an erbium-doped fiber laser to the standard quantum limit by intracavity spectral filtering
}

\author{
Steve Sanders, Namkyoo Park, Jay W. Dawson, and Kerry J. Vahala \\ Department of Applied Physics, 128-95, California Institute of Technology, \\ Pasadena, California 91125
}

(Received 5 June 1992; accepted for publication 5 August 1992)

The high frequency intensity noise of a tandem fiber Fabry-Perot erbium-doped fiber ring laser is reduced to the standard quantum limit, with a $0.5 \mathrm{~dB}$ experimental uncertainty. Noise reduction of $>14 \mathrm{~dB}$ is achieved by intracavity spectral filtering of weak side modes using a narrow-band fiber Fabry-Perot etalon.

Broadly tunable, single-frequency erbium-doped fiber lasers have recently, been demonstrated and are potential sources for systems operating near $1.5 \mu \mathrm{m} .{ }^{1,2}$ Measurements of an erbium-doped fiber laser using tandem fiber Fabry-Perot (FFP) filters for mode selection revealed that the intensity noise at high frequency $(310 \mathrm{MHz})$ depended linearly upon laser power, had a resonance structure with the $4 \mathrm{MHz}$ free spectral range of the laser, and could be maintained within $20 \mathrm{~dB}$ of the standard quantum limit throughout a $24 \mathrm{~nm}$ tuning range. ${ }^{3}$ This excess noise power was attributed to the beating of the lasing mode with strongly damped side modes. In this letter, we present an analysis of this side mode beat noise in order to show how it may be reduced in optimized cavity configurations. We then demonstrate noise levels within experimental uncertainty of the standard quantum limit (SQL), by implementing intracavity spectral filtering combined with lower cavity loss.
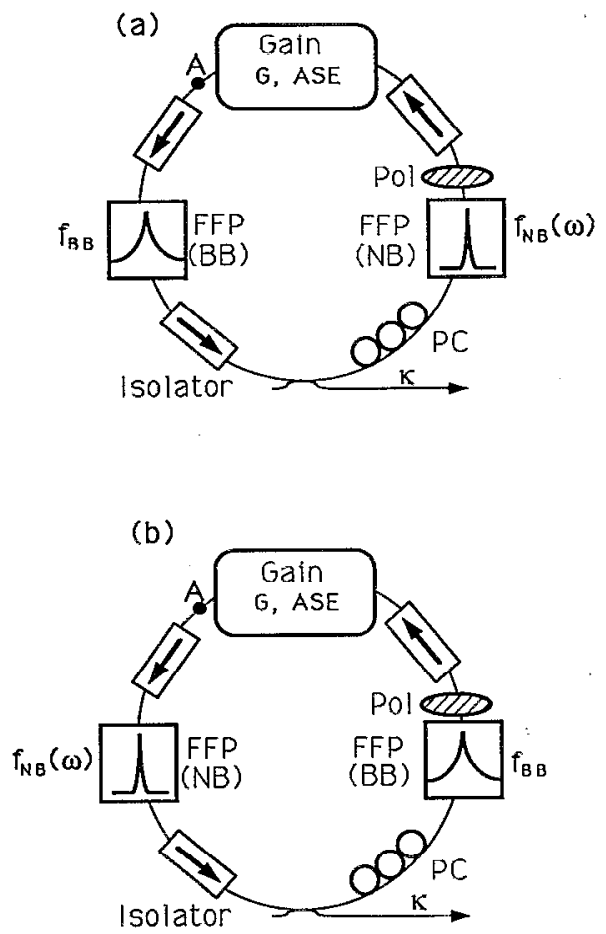

FIG. 1. The tandem fiber Fabry-Perot erbium ring laser in configuration (a) with the narrow-band FFP before the gain medium to increase output power and (b) with the narrow-band FFP after the gain medium to filter side modes (PC:polarization controller; FFP: fiber Fabry-Perot filter).
Consider the two configurations for a tandem fiber Fabry-Perot (FFP) erbium-doped ring laser shown in Fig. 1, where the narrow band FFP, with $130 \mathrm{MHz}$ full width at half-maximum (FWHM) transmission and $10.2 \mathrm{GHz}$ free-spectral range (FSR), selects an individual lasing mode, and the broadband ( $38 \mathrm{GHz}$ FWHM, $4020 \mathrm{GHz}$ FSR) FFP tunes the lasing wavelength over the erbium gain spectrum. In one pass, the gain medium amplifies the optical power by a factor $G$ and adds amplified spontaneous emission (ASE) power with a spectral density $\tilde{n}_{\mathrm{sp}}(G$ $-1) \hbar \omega$, where $\omega$ is the optical frequency and $\tilde{n}_{\mathrm{sp}}$ is the integrated spontaneous emission factor defined in Ref. 4 and given by

$$
\tilde{n}_{\mathrm{sp}}=\frac{G}{G-1} \int_{0}^{l} d z g(z) n_{\mathrm{sp}}(z) e^{-\int_{0}^{z} g\left(z^{\prime}\right) d z^{\prime}},
$$

where $g(z)=\sigma_{e} N_{2}(z)-\sigma_{a} N_{1}(z)$ and $n_{\mathrm{sp}}(z)=\sigma_{e} N_{2}(z) /$ $g(z) ; \sigma_{e}$ and $\sigma_{a}$ are the emission and absorption cross sections, respectively, and $N_{2}$ and $N_{1}$ are the upper and lower state population densities, respectively. (When $n_{\mathrm{sp}}$ is independent of $z, \tilde{n}_{\mathrm{sp}}=n_{\mathrm{sp}}$.) The FFP filters have intensity transfer functions $f_{N B}(\omega)$ and $f_{B B}$, where the frequency dependence of the broad-band filter can be neglected in determining the side mode powers. For both configurations, the steady-state electric field of the weak side modes

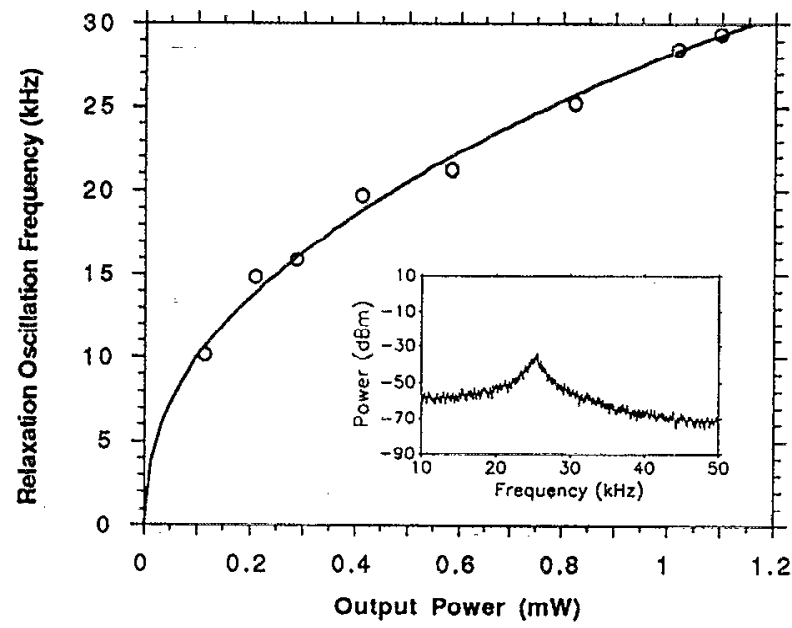

FIG. 2. Relaxation resonance frequency vs laser output power ( $50 \%$ output coupler). Inset: Intensity noise power vs frequency for $820 \mu \mathrm{W}$ laser output power ( $35 \mathrm{~dB}$ amplifier, $1 \mathrm{kHz}$ resolution). 


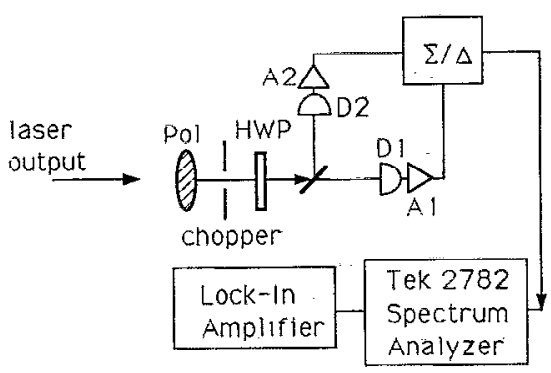

FIG. 3. Balanced homodyne detection system to determine laser noise level relative to the SQL (shot noise floor). The SQL is measured when the photocurrents are differenced; the laser noise is measured when the photocurrents are summed. (HWP: half-wave plate; D1 and D2: BT\&D PDH0004 detectors; A1 and A2: Avantek ACT10-213-1 amplifiers).

immediately after the gain medium (at point $A$ ) has an optical power spectral density:

$$
\left|\widetilde{E}_{A}(\omega)\right|^{2}=\frac{\tilde{n}_{\mathrm{sp}}(G-1) \hbar \omega}{1-2 \gamma \cos \left(k l_{c}\right)+\gamma^{2}},
$$

where $l_{c}$ is the length of the fiber laser cavity; $k=(n \omega) / c$, and $\gamma^{2}$ is the net round-trip power gain, given by

$$
\gamma^{2}(\omega)=f_{N B}(\omega)(1-\kappa) f_{B B} G(1-\epsilon),
$$

$\kappa$ is the power output coupling and $\epsilon$ is the round-trip loss due to other cavity components.

The intensity noise power spectral density outside the laser ring arising from beating between the lasing mode at frequency $\omega_{0}$, with output power $\kappa f\left(\omega_{0}\right) E_{0}^{2}$, and amplified spontaneous emission at frequencies $\omega_{0}+\Omega$ and $\omega_{0}-\Omega$, with optical output power spectral densities $\kappa f\left(\omega_{0}\right.$ $+\Omega) \widetilde{E}_{+}^{2}(\Omega)$ and $\kappa f\left(\omega_{0}-\Omega\right) \widetilde{E}_{-}^{2}(-\Omega)$, respectively, is

$$
\begin{aligned}
W_{P P}(\Omega)= & 2 E_{0}^{2}\left[\widetilde{E}_{+}^{2}(\Omega)+\widetilde{E}_{-}^{2}(-\Omega)\right] \kappa^{2} f\left(\omega_{0}\right. \\
& +\Omega) f\left(\omega_{0}\right),
\end{aligned}
$$

where $E_{0}^{2}$ is the optical power in the lasing mode at point $A$, and $\widetilde{E}_{+}^{2}$ and $\widetilde{E}_{-}^{2}$ are the optical power spectral densities at point $A$ of the amplified spontaneous emission at frequency offsets of $\Omega$ and $-\Omega$ from the lasing mode, respectively. The filter response $f$ represents $f_{B B}$ for configuration (a)

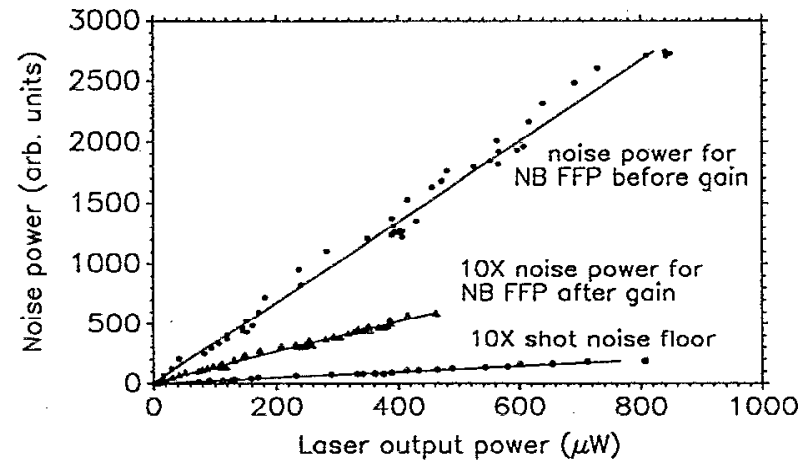

FIG. 4. Laser noise power at $307.5 \mathrm{MHz}$ with $90 \%$ output coupler and narrow-band FFP before gain medium (upper trace) and narrow-band FFP between gain medium and output coupler (middle trace.) The noise power in the middle trace and the SQL are multiplied by 10 . and represents $f_{N B}$ for configuration (b) in Fig. 1, and a small asymmetry that may arise in $f_{N B}(\omega)$ about $\omega_{0}$ due to detuning of the lasing mode from the narrow-band FFP transmission peak has been neglected. Using Eq. (2) and normalizing to the shot noise power spectrum of $2 \kappa f\left(\omega_{0}\right) E_{0}^{2} \hbar \omega$ gives

(a) $90 \%$ output coupling

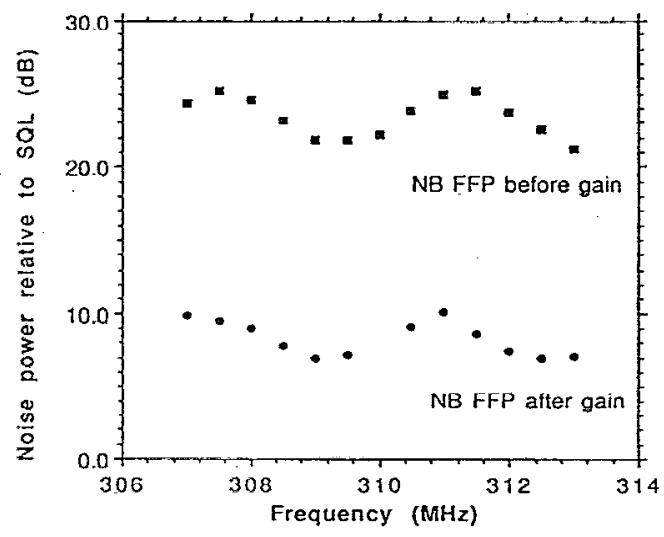

(b) $50 \%$ output coupling

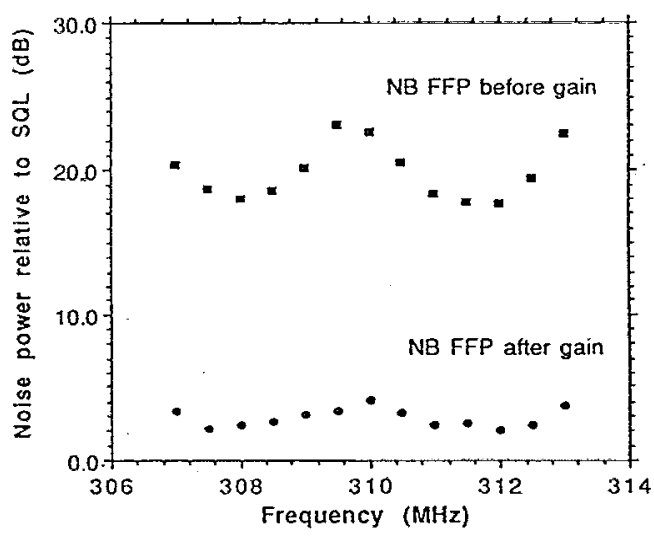

(c) $10 \%$ output coupling

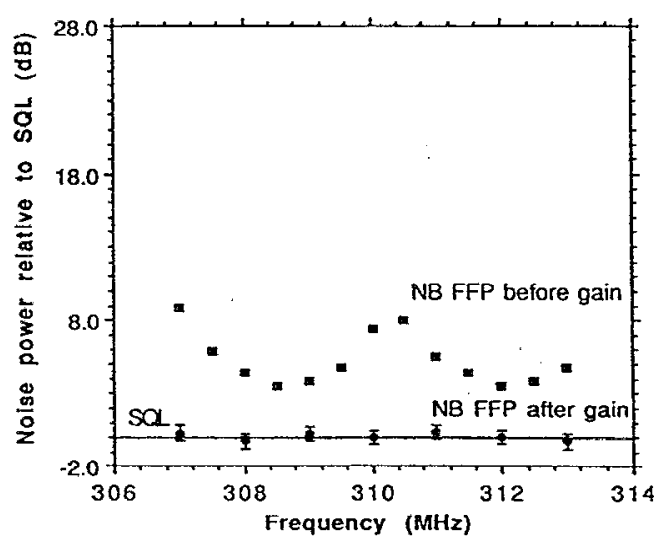

FIG. 5. Laser noise power spectra relative to $\mathrm{SQL}$ around $310 \mathrm{MHz}$ for (a) $90 \%$ output coupling, (b) $50 \%$ output coupling, and (c) $10 \%$ output coupling. With the narrow-band FFP after the gain medium, the intensity noise power reaches the SQL for $10 \%$ output coupling. 


$$
\frac{W_{P P}}{W_{\mathrm{SQL}}}(\Omega)=\frac{2 \tilde{n}_{\mathrm{sp}}(G-1) \kappa f\left(\omega_{0}+\Omega\right)}{1-2 \gamma \cos \left[\left(n \Omega l_{c}\right) / c\right]+\gamma^{2}}
$$

A resonance structure of the intensity noise power, as discussed in Ref. 3 and shown in Fig. 5, is apparent from the denominator of Eq. (5). The average noise level over a free spectral range of the fiber laser is determined by $\widetilde{n}_{\mathrm{sp}}$, the single pass gain, the output coupling, and the transfer function of the FFP filter after the gain medium. The noise power can be significantly reduced by placing the narrowband FFP between the gain medium and the output coupler and by reducing the output coupling to lower the single pass gain. An analysis of the spatial variation of the inversion is required to determine $\widetilde{n}_{\mathrm{sp}}{ }^{4,5}$ and it is expected to increase as the inversion at threshold is reduced by lowering the output coupling.

If the noise from beating of the lasing mode and side modes can be adequately reduced, then shot noise in the lasing mode will be the dominant source of intensity noise, and the total intensity noise level will approach the standard quantum limit. Excess noise due to inversion dynamics and pumping is expected to be negligible at high frequencies, due to the low relaxation resonance frequency of the erbium laser. Figure 2 shows the measured relaxation resonance spectrum near $25 \mathrm{kHz}$ for an $820 \mu \mathrm{W}$ laser output power and that this resonance frequency occurs in the tens of kilohertz, increasing as the square root of the laser power, in accordance with theory. ${ }^{6}$

Measurements of the SQL and laser intensity noise power versus laser output power are performed using the balanced homodyne detection (BHD) system shown in Fig. 3, balanced to give a common-mode rejection $>40 \mathrm{~dB}$. The laser gain medium used throughout the experiments is a Corning Fibergain module with $20 \mathrm{~m}$ of $\mathrm{Er}^{+3}$-doped fiber co-doped with aluminum; the amplifier pump beam copropagates with the lasing mode. The laser intensity noise power at $307.5 \mathrm{MHz}$ and SQL measurements as a function of optical power at the ring output are shown in Fig. 4 for $90 \%$ laser output coupling and the two configurations of Fig. 1. With the narrow-band FFP placed between the gain medium and output coupler, as in Fig. 1(b), a $15 \mathrm{~dB}$ noise reduction is observed compared with configuration (a). This reduction is comparable to the $17 \mathrm{~dB}$ of additional filtering provided by the narrow-band FFP, as compared to the broadband FFP at $310 \mathrm{MHz}$. Because the narrow-band FFP has $3 \mathrm{~dB}$ higher insertion loss at the transmission peak than the broad-band FFP, the laser output power is
TABLE I. Power at output of laser ring for configurations: (a) narrowband FFP before gain medium and (b) narrow-band FFP between gain medium and output coupler.

\begin{tabular}{ccrc}
\hline Output coupling & Pump laser current & Power (a) & Power (b) \\
\hline $90 \%$ & $150 \mathrm{~mA}$ & $710 \mu \mathrm{W}$ & $450 \mu \mathrm{W}$ \\
$50 \%$ & $250 \mathrm{~mA}$ & $1230 \mu \mathrm{W}$ & $730 \mu \mathrm{W}$ \\
$10 \%$ & $300 \mathrm{~mA}$ & $360 \mu \mathrm{W}$ & $200 \mu \mathrm{W}$ \\
\hline \hline
\end{tabular}

slightly reduced for the lower noise configuration, as shown in Table I.

The intensity noise power near $310 \mathrm{MHz}$ relative to the SQL is shown in Fig. 5 for output couplings of $90 \%, 50 \%$, and $10 \%$, with both configurations (a) and (b) of Fig. 1. Over a $6 \mathrm{MHz}$ frequency span, $14-16$ and $16-20 \mathrm{~dB}$ reductions in noise power are observed in changing from configuration (a) to configuration (b) for the $90 \%$ and $50 \%$ output couplings, respectively. The intensity noise power actually reaches the SQL in configuration (b) with the $10 \%$ output coupler. All of the data shown in Fig. 5 have been corrected, as described in Ref. 3, to give the noise power at the fiber laser output by accounting for the $40 \%$ to $48 \%$ total measurement quantum efficiencies, which include both the detector efficiencies and losses before the BHD system. Experimental uncertainty in the lowest noise measurement is $0.2 \mathrm{~dB}$, which increases to $0.5 \mathrm{~dB}$ when corrected for the optical losses.

We have shown how the high frequency intensity noise arising from side modes in an erbium-doped tandem FFP fiber laser can be greatly suppressed by intracavity spectral filtering between the gain medium and the laser output. By employing this technique in a laser with reduced output coupling losses, intensity noise was reduced to the standard quantum limit.

This work was supported by the Office of Naval Research, Contract No. N00014-91-J-1524, and Northrop Corporation.

'N. Park, J. W. Dawson, K. J. Vahala, and C. Miller, Appl. Phys. Lett. 59, 2369 (1991).

${ }^{2}$ J. L. Zyskind, J. W. Sulhoff, Y. Sun, J. Stone, L. W. Stulz, G. T. Harvey, D. J. DiGiovanni, H. M. Presby, A. Piccirilli, U. Koren, and R. M. Jopson, Electron. Lett. 27, 2148 (1991).

${ }^{3}$ S. Sanders, J. W. Dawson, N. Park, and K. J. Vahala, Appl. Phys. Lett. 60, 2583 (1992).

${ }^{4}$ E. Desurvire, IEEE Photon. Technol. Lett. 2, 208 (1990).

${ }^{5}$ R. Olshansky, Electron. Lett. 24, 1363 (1988).

"A. E. Siegman, Lasers (University Science Books, Mill Valley, CA, 1986), pp. 962-964. 